\title{
Familial aggregation of stillbirth: a pedigree analysis of a matched case control study
}

Tsegaselassie Workalemahu ${ }^{1}$, Jessica Page ${ }^{2}$, Huong Meeks ${ }^{3}$, Zhe Yu ${ }^{3}$, Emily Guinto ${ }^{3}$, Alison Fraser ${ }^{3}$, Michael Varner ${ }^{1}$, Lauren Theilen ${ }^{1}$, Aaron Quinlan ${ }^{1}$, Hilary Coon ${ }^{1}$, Daniel Enquobahrie $^{4}$, Cande Ananth (STATS CONSULTS ONLY) ${ }^{5}$, Fasil Tekola-Ayele ${ }^{6}$, Lynn Jorde $^{1}$, and Robert Silver (USA) ${ }^{1}$

${ }^{1}$ University of Utah Health

${ }^{2}$ Intermountain Medical Center

${ }^{3}$ University of Utah Health Huntsman Cancer Institute

${ }^{4}$ University of Washington

${ }^{5}$ Rutgers Robert Wood Johnson Medical School

${ }^{6}$ Eunice Kennedy Shriver National Institute of Child Health and Human Development

February 19, 2022

\begin{abstract}
Objective To determine if stillbirth aggregates in families and quantify its familial risk using extended pedigrees. Design Statewide matched case-control study. Setting Utah, United States. Population Stillbirth cases (n=9 404) and live-birth controls (18 808) between 1978 and 2019. Methods Using the Utah Population Database, a population-based genealogical resource linked with state fetal death and birth records, we identified high-risk pedigrees with excess familial aggregation of stillbirth using the Familial Standardized Incidence Ratio (FSIR). Stillbirth odds ratio (OR) for first-degree relatives (FDR), seconddegree relatives (SDR), and third-degree relatives (TDR) of parents with a stillbirth and live-birth were estimated using logistic regression models. Results We identified 390 high-risk pedigrees with evidence for excess familial aggregation (FSIR[?]2.00 and P-value $<0.05)$. FDRs, SDRs and TDRs of affected parents had 1.14-fold (95\% confidence interval [CI]: 1.04-1.26), 1.22-fold (95\% CI: 1.11-1.33), and 1.15-fold (95\% CI: 1.08-1.21) higher stillbirth odds compared to FDRs, SDRs and TDRs of unaffected parents, respectively. Parental sex-specific analyses showed male FDRs, SDRs and TDRs of affected fathers had 1.22-fold (95\% CI: 1.02-1.47), 1.38-fold (95\% CI: 1.17-1.62), 1.17-fold (95\% CI: 1.05-1.30) higher stillbirth odds compared to those of unaffected fathers, respectively. FDRs, SDRs and TDRs of affected mothers had 1.12-fold (95\% CI: 0.98-1.28), 1.09-fold (95\% CI: 0.96-1.24), and 1.15-fold (95\% CI: 1.06-1.24) higher stillbirth odds compared with those of unaffected mothers, respectively. Conclusions We provide evidence for familial aggregation of stillbirth. Our findings warrant investigation into genes associated with stillbirth and underscore the need to design large-scale studies to determine its genetic architecture.
\end{abstract}

\section{Familial aggregation of stillbirth: a pedigree analysis of a matched case control study}

Tsegaselassie Workalemahu, PhD, MS*1; Jessica M Page, MD, MSCI*1,2 ; Huong Meeks, PhD, MS ${ }^{3}$; Zhe Yu, $\mathrm{MS}^{3}$; Emily Guinto, $\mathrm{MS}^{3}$; Alison Fraser, $\mathrm{MSPH}^{3}$; Michael W. Varner, $\mathrm{MD}^{1,2}$; Lauren Theilen, MD, MSCI${ }^{1}$; Aaron Quinlan, $\mathrm{PhD}^{4}$; Hilary Coon, $\mathrm{PhD}^{5}$; Daniel A Enquobahrie, $\mathrm{MD}, \mathrm{PhD}, \mathrm{MPH}^{6}$; Cande V. Ananth, $\mathrm{PhD}, \mathrm{MPH}^{7,8}$; Fasil Tekola-Ayele, $\mathrm{PhD}, \mathrm{MPH}^{9}$; Lynn B. Jorde, $\mathrm{PhD}, \mathrm{MS}^{4}$; Robert M. Silver, $\mathrm{MD}^{1,2}$

\section{Affiliations :}

${ }^{1}$ University of Utah Health, Department of Obstetrics and Gynecology, Salt Lake City, UT; 
${ }^{2}$ Intermountain Healthcare, Maternal-Fetal Medicine, Salt Lake City, UT;

${ }^{3}$ Population Sciences, Huntsman Cancer Institute, University of Utah School of Medicine, Salt Lake City, $\mathrm{UT}$;

${ }^{4}$ University of Utah, Department of Human Genetics, Salt Lake City, UT;

${ }^{5}$ University of Utah, Department of Psychiatry and Huntsman Mental Health Institute, Salt Lake City, UT;

${ }^{6}$ Department of Epidemiology, University of Washington, Seattle, WA;

${ }^{7}$ Division of Epidemiology and Biostatistics. Rutgers, The State University of New Jersey, NJ;

${ }^{8}$ Department of Obstetrics, Gynecology, and Reproductive Sciences, New Brunswick, NJ;

${ }^{9}$ Epidemiology Branch, Division of Population Health Research, Eunice Kennedy Shriver National Institute of Child Health and Human Development, National Institutes of Health, Bethesda MD

Corresponding author : Tsegaselassie Workalemahu, PhD MS; University of Utah, 30 North 1900 East, Suite 2B293, Salt Lake City, UT, 84132. Phone: 801-587-1607. Email: tsegaselassie.workalemahu@hsc.utah.edu

This work was supported by grant funding from the National Center for Advancing Translational Sciences of the National Institutes of Health (grant 1UL01TR002538). The content is solely the responsibility of the authors and does not necessarily represent the official views of the National Institutes of Health. Author TW is supported by the National Center for Advancing Translational Sciences of the National Institutes of Health (KL2 grant TR002549). Author CVA is supported, in part, by the National Heart, Lung, and Blood Institute (grant R01-HL150065) and the National Institute of Environmental Health Sciences (grant R01-ES033190). Author LBJ is supported by National Institute of General Medical Sciences (grant R35GM118335).

\section{Abstract \\ Objective}

To determine if stillbirth aggregates in families and quantify its familial risk using extended pedigrees.

Design

State-wide matched case-control study.

\section{Setting}

Utah, United States.

\section{Population}

Stillbirth cases (n=9 404) and live-birth controls (18 808) between 1978 and 2019.

\section{Methods}

Using the Utah Population Database, a population-based genealogical resource linked with state fetal death and birth records, we identified high-risk pedigrees with excess familial aggregation of stillbirth using the Familial Standardized Incidence Ratio (FSIR). Stillbirth odds ratio (OR) for first-degree relatives (FDR), second-degree relatives (SDR), and third-degree relatives (TDR) of parents with a stillbirth and live-birth were estimated using logistic regression models.

\section{Results}

We identified 390 high-risk pedigrees with evidence for excess familial aggregation (FSIR[?]2.00 and Pvalue $<0.05)$. FDRs, SDRs and TDRs of affected parents had 1.14-fold (95\% confidence interval [CI]: 1.041.26), 1.22-fold (95\% CI: 1.11-1.33), and 1.15-fold (95\% CI: 1.08-1.21) higher stillbirth odds compared to FDRs, SDRs and TDRs of unaffected parents, respectively. Parental sex-specific analyses showed male FDRs, 
SDRs and TDRs of affected fathers had 1.22-fold (95\% CI: 1.02-1.47), 1.38-fold (95\% CI: 1.17-1.62), 1.17-fold (95\% CI: 1.05-1.30) higher stillbirth odds compared to those of unaffected fathers, respectively. FDRs, SDRs and TDRs of affected mothers had 1.12-fold (95\% CI: 0.98-1.28), 1.09-fold (95\% CI: 0.96-1.24), and 1.15-fold (95\% CI: 1.06-1.24) higher stillbirth odds compared with those of unaffected mothers, respectively.

\section{Conclusions}

We provide evidence for familial aggregation of stillbirth. Our findings warrant investigation into genes associated with stillbirth and underscore the need to design large-scale studies to determine its genetic architecture.

\section{Keywords}

Stillbirth; familial; genetics; pedigree

\section{Running title}

Familial aggregation of stillbirth

\section{Tweetable abstract}

Stillbirth aggregates in families and the risk of stillbirth for parents with a family history was greater than those without a family history.

\section{Introduction}

Stillbirth is common worldwide with rates exceeding the neonatal and infant death rates in many high-income countries. ${ }^{1}$ This is driven in part by a large number of unexplained stillbirths. In a well characterized cohort of stillbirth cases (fetal death at [?]20 weeks' gestation) in the U.S., approximately $24 \%$ of stillbirths remained unexplained following thorough, standardized evaluation. ${ }^{2}$ The identification of stillbirths due to genetic abnormalities has improved with advancing genomic technologies. The addition of microarray improved the diagnostic yield from $6.5 \%$ to $8.8 \%$ as compared to karyotype. ${ }^{3}$ Moreover, $8.5 \%$ of stillbirth cases with a normal chromosomal microarray had a probable molecular genetic diagnosis attributable to Mendelian disorders discovered on whole exome sequencing. ${ }^{4}$ The combined results of karyotype, microarray and whole exome sequencing identified a potential genetic cause of death in $18 \%$ of stillbirth cases. ${ }^{4}$ These observations highlight the possibility of a heritable component in the genesis of stillbirth.

Adverse pregnancy outcomes, particularly ischemic placental disease (preeclampsia, placental abruption and small for gestational age) and preterm birth, aggregate in families. ${ }^{5-13}$ These data suggest that there are genetic underpinnings for many adverse pregnancy outcomes. However, data regarding an inherited genetic risk for stillbirth are limited. One study evaluated mother-daughter pairs and did not find an inherited predisposition for stillbirth. ${ }^{14}$ Given that there are both maternal and paternal contributions to the fetal genotype, investigation into broader pedigrees may reveal important insights to understand the extent to which genes may affect stillbirth risk. ${ }^{15}$ Thus, we sought to investigate whether stillbirth aggregates in families and, if so, to quantify the risk of stillbirth for parents with family history in extended pedigrees.

\section{Methods}

\section{Study design and population}

We conducted an extended pedigree analysis of stillbirth using the Utah Population Database (UPDB). The UPDB contains genealogy information linked to Utah fetal death and live birth certificates, and hospital records databases. Request for permission to use family structure data was approved by the Utah Resource for Genetic and Epidemiologic Research (RGE), the UPDB data use oversight committee. This study was approved by the Institutional Review Board of the University of Utah.

The UPDB genealogy record extends up to as many as 17 generations. It also includes approximately 5.8 million individuals with family relationship data dating back at least 3 generations. ${ }^{16}$ Stillbirth cases were identified using electronic Utah fetal death records that were available between 1978 and 2019. We used 
previously suggested nomenclature for stillbirth, defined as the birth of an infant with no signs of life (Apgar scores of 0 and 0 ) at [?] 20 weeks' gestation. ${ }^{17}$ Gestational age was used as reported on birth and fetal death certificates. The 1978 revision of the birth and fetal death certificates used the last menstrual period (LMP) for gestational age determination with addition of a correction based on the physician's judgement if the LMP was unknown or incorrect. ${ }^{18}$ This system was utilized until the 2003 revision, when the National Center for Health Statistics utilized the best obstetric estimate for gestational age reporting on vital statistics records. This method uses the last menstrual period dating with correction as needed if estimated gestational age by ultrasound differs significantly from menstrual dates. ${ }^{19}$ Controls were identified as singleton live birth(s) and without reports of stillbirth, miscarriage, or pregnancy termination (as indicated in the Utah birth certificates). Controls were 2:1 matched to cases based on maternal age at stillbirth/ delivery, stillbirth or live birth-year, and minimum pedigree information (those with informative vs uninformative relationship data in their pedigrees). ${ }^{20}$ Extended pedigrees of parents of stillbirth cases and controls included biological first-degree relatives (FDR; parents, full-siblings, and children), second-degree relatives (SDR; grandparents, full aunts/uncles, nieces/nephews, and half-siblings) and third-degree relatives (TDR; cousins from full aunts/uncles, grandnieces/ grandnephews from full siblings, great aunts/uncles, great grandchildren, and half-nieces/ nephews from half-siblings). The inclusion criteria for cases, controls, and parent's relatives were based on maternal age 13-40 years old and paternal age [?]13 years old between 1978 and 2019, and knowledge of offspring and parental sex. We restricted records to 13-40 years old mothers at the time of offspring death/birth-year, because maternal age $>40$ years is strongly associated with unexplained stillbirth. ${ }^{21,22}$

\section{Statistical analysis}

Statistical analyses included all available stillbirth cases $(n=9,404)$, controls $(n=18,808)$ that were 2:1 matched with cases, and relatives of the parents of cases and controls. We compared maternal demographic characteristics of stillbirth cases to those of live birth controls using t-tests for continuous variables and chi-square tests for categorical variables.

\section{Determination of stillbirth familial aggregation}

We used the genealogical index, ${ }^{6}$ which considers all relationships in the UPDB, to identify pedigrees of stillbirth index cases (i.e., probands). To capture the maternally and paternally inherited risk of stillbirth, we designated the offspring rather than mothers as probands. We estimated the Familial Standardized Incidence Ratio (FSIR) to determine the level of stillbirth familial aggregation and identify high-risk pedigrees. ${ }^{23}$ The FSIR compares the observed incidence of stillbirth in a pedigree to its expected incidence in the UPDB population, weighting the familial risk contribution of each relative in a pedigree by the kinship coefficient (i.e., the probability that a relative shares an allele with the proband through a common ancestor). ${ }^{24}$ Our $a$ priorispecified criteria for familial aggregation and a high-risk pedigree were pedigrees with FSIR [?]2 and Pvalue $<0.05 .{ }^{23}$ We evaluated the skewness of FSIR among high-risk pedigrees by examining its distribution. We presented the top 10 high-risk pedigrees sorted by their FSIR and founders to eliminate any multiple founders claiming the same descendants through marriage. ${ }^{25}$ To avoid potential biased estimates of stillbirth familial aggregation due to multiple partnerships, we excluded multiple partnerships.

\subsection{Determination of stillbirth risk among relatives}

We used logistic regression models to estimate the odds ratio (95\% confidence interval [95\%: CI]) of stillbirth, comparing the odds of stillbirth in relatives of parents of stillbirth cases to the odds of stillbirth in relatives of parents of live birth controls. Estimates were adjusted for birth year, race (whether non-Hispanic White), and ethnicity (whether Hispanic). Case-control statuses of FDRs, SDRs and TDRs of affected and unaffected parents were similarly ascertained using identical case and control definitions in probands. To identify distinct parent-of-origin effects on stillbirth, we conducted parental sex-specific analyses. Statistical analyses were carried out using SAS (version 9.4, SAS Institute Inc., Cary, NC), R (version 4.0.2), and STATA version 15.0 (StataCorp, College Station, TX).

\section{Results}




\section{Study population}

We identified probands (stillbirth cases; $\mathrm{n}=9,404)$ and their parents' FDRs $(\mathrm{n}=73,339)$, SDRs $(\mathrm{n}=128,083)$ and TDRs $(\mathrm{n}=300,536)$, as well as probands (live birth controls; $\mathrm{n}=18,808$ ) and their parents' FDRs $(\mathrm{n}=166,188)$, SDRs $(\mathrm{n}=258,103)$ and TDRs $(\mathrm{n}=608,803)$ (Figure S1and Table S1 $)$. The rate of stillbirth among 1,903,782 births that occurred between 1978 and 2019 in the state of Utah was $0.5 \%$, comparable rate to that of high-income countries $(0.6 \%) .{ }^{1}$ The proportion of male infant sex slightly differed between cases $(\mathrm{n}=4,735 ; 50.4 \%)$ and controls $(\mathrm{n}=9,778 ; 52.0 \%)$. The median (IQR) birth weight in grams was 1,145 $(440-2,567)$ in cases and 3,340 (3,020-3,646) in controls. The median (IQR) gestational ages of cases and controls were 29 (23-36) and 39 (38-40) weeks, respectively. The study population was comprised mostly of non-Hispanic Whites (66.0\% cases and $73.0 \%$ controls). There was higher representation of non-Hispanic Blacks (0.7\% vs $0.3 \%)$ and Hispanics $(24.5 \%$ vs $21.2 \%$ ) in cases than controls, respectively. Paternal demographic factors followed a similar pattern with a lower proportion of non-Hispanic Whites (60.6\% vs 69.7\%) and higher numbers of non-Hispanic Blacks ( $0.8 \%$ vs $0.5 \%$ ) in cases compared to controls, respectively. Cases had lower maternal educational attainment (post-college $4.5 \%$ vs $7.1 \%$ ), fewer prenatal visits (9 vs 11) and slightly higher pre-pregnancy body-mass-index (25.7 vs $\left.24.7 \mathrm{~kg} / \mathrm{m}^{2}\right)$ than controls. Maternal comorbidities were more common among cases than controls, with higher rates of chronic hypertension $(1.0 \%$ vs $0.6 \%)$, hypertensive disorder of pregnancy $(12.4 \%$ vs $9.9 \%)$ and pregestational diabetes ( $1.6 \%$ vs $0.6 \%)$. Major congenital anomalies of the fetus were more common among cases than controls $(7.3 \%$ vs. $0.8 \%)$.

\section{Familial aggregation of stillbirth}

Among stillbirth cases, we identified 2,498 (26.6\%) probands with high-risk pedigrees $(\mathrm{n}=390)$, showing evidence for excess stillbirth familial aggregation (FSIR [?]2.00 and P-value $<0.05$ ) in the UPDB population (Table 1 ). Among high-risk pedigrees, the FSIR values ranged from 2.00 to 7.54 (Figure S2 ), indicating that the aggregation of stillbirth in the pedigrees is 2-8 times greater than what we would expect in the UPDB population by chance. A representative high-risk pedigree (pedigree \#3 in Table 1 ) displayed in Figure 1 shows 10 stillbirth cases that descended from a common founder.

\section{Stillbirth risk among relatives}

FDRs, SDRs and TDRs of affected parents had 1.14-fold (95\% CI: 1.04-1.26), 1.22-fold (95\% CI: 1.11-1.33), and 1.15-fold (95\% CI: 1.08-1.21) higher stillbirth odds compared with FDRs, SDRs and TDRs of unaffected parents, respectively (Figure 2 ). In parental sex-stratified analyses, FDRs, SDRs and TDRs of affected fathers had 1.17-fold (95\% CI: 1.02-1.34), 1.34-fold (95\% CI: 1.19-1.51), and 1.14-fold (95\% CI: 1.06-1.24) higher stillbirth odds compared with those of unaffected fathers, respectively (Table 2 ). Similarly, FDRs, SDRs and TDRs of affected mothers had 1.12-fold (95\% CI: 0.98-1.28), 1.09-fold (95\% CI: 0.96-1.24), and 1.15-fold (95\% CI: 1.06-1.24) higher stillbirth odds compared with those of unaffected mothers, respectively. Furthermore, stillbirth odds was pronounced among male relatives, where the corresponding OR (95\% CI) for male FDRs, SDRs and TDRs of affected fathers was 1.22 (1.02-1.47), 1.38 (1.17-1.62), and 1.17 (1.051.30), whereas the corresponding OR (95\% CI) for male FDRs, SDRs and TDRs of affected mothers was 1.05 (0.86-1.28), 1.19 (0.99-1.42), and 1.17 (1.05-1.31). Lastly, the OR (95\% CI) for female relatives of affected fathers was $1.10(0.90-1.35), 1.31(1.10-1.57)$, and $1.12(1.00-1.25)$, and the OR (95\% CI) for female relatives of affected mothers was 1.19 (0.98-1.43), 1.01 (0.84-1.21), and 1.12 (1.00-1.25), respectively.

\section{Discussion}

Our work demonstrates that there is familial aggregation of stillbirth, implying that a family history of stillbirth imparts increased stillbirth risk to family members. Stillbirth risk was stronger in the male relatives of affected pedigrees. Familial aggregation of stillbirth supports existing data regarding genetic underpinnings of stillbirth. ${ }^{26}$ Identification of stillbirth high-risk pedigrees of may lead to improved characterization of these families, causal genes and extreme phenotypes such as fetal death.

There are limited data on familial aggregation of fetal death. Some of the difficulty in studying fetal death lies in varying definitions of miscarriage, fetal death and stillbirth worldwide. ${ }^{17}$ Additionally, many studies 
have relied on self-reported outcomes or chart abstraction, increasing the risk of inaccuracies and recall bias. Accordingly, data regarding early pregnancy loss have been mixed in regard to a heritable risk of spontaneous abortion or fetal death..$^{27,28}$ Recent studies by Woolner and colleagues investigated familial risk of miscarriage ${ }^{29}$ or stillbirth ${ }^{14}$ in mother-daughter pairs. Both studies included mothers (pregnancies between 1949-2000) with at least one daughter (pregnancies between 1965-2016) in Aberdeen, United Kingdom. The study of miscarriage included 31,565 mother-daughter pairs and the study of stillbirth included 27,688 mother-daughter pairs. They demonstrated an increased risk of miscarriage but not stillbirth in their intergenerational analysis. ${ }^{14,29}$ This difference between our results and theirs may be due to several factors. First, an intergenerational link may have been missed in their work due to the relative rarity of fetal death [?]24 weeks and limiting the analysis to mother-daughter pairs. By utilizing the UPDB, we were able to evaluate multigenerational trends in fetal death as well as both maternal and paternal lineages. This likely increased our ability to detect a familial aggregation for this relatively uncommon pregnancy outcome. Second, they defined miscarriage as fetal death prior to 24 weeks and stillbirth as fetal death at 24 weeks and greater. This differs from our definition of stillbirth as fetal death at 20 weeks or greater. Their study likely captured many fetal death cases (16 - 23 weeks) which share pathophysiologic characteristics of fetal death at 20 weeks and beyond. ${ }^{17}$ Indeed, the median (IQR) gestational age in weeks among women with high-risk stillbirth FSIR was lower (29 [23-26]) compared with all affected women (39 [38-40]) included in our study.

By utilizing the stillborn fetus as the proband we were able to assess both maternal and paternal contributions to the fetal death phenotype. We demonstrated a stronger risk of stillbirth in male relatives of affected pedigrees as compared to female descendants. This risk was further increased if a paternal history of stillbirth was present. Paternal genes may play a key role in placentation, a critical process for fetal development. ${ }^{15}$ Esplin and colleagues demonstrated the importance of paternal genetic contribution in preeclampsia, a process believed to have pathologic placental underpinnings. ${ }^{13}$ The genetic basis for stillbirth is as yet poorly characterized and our work highlights an opportunity to explore parental sex-specific differences in stillbirth familial risk.

Our findings demonstrated an elevated risk of stillbirth for parents with any family history of stillbirth. This risk persisted into TDR, suggesting that there may be inherited genetic factors that impart an increased risk of stillbirth that are independent of shared environmental characteristics. However, gene-environment interactions are complex and epigenetic modifications as a result of environmental exposures can be inherited and affect the health outcomes of future generations. ${ }^{30}$ Evaluating the inherited pathogenic genes in these families will improve our understanding of this process.

There are many pathophysiologic mechanisms by which genetic abnormalities may lead to stillbirth. Genetic changes resulting in placental insufficiency, severe fetal growth restriction, anatomic anomalies not readily identified by ultrasound or metabolic derangements have been proposed as causative or contributory. ${ }^{31}$ Stanley and colleagues performed whole exome sequencing in a large stillbirth case cohort and identified previously unknown genetic variants leading to a suspected genetic cause of death in $18 \%$ of the stillbirths in the study cohort. ${ }^{4}$ They found variants in genes that are known to be pathogenic in postnatal life but also variants that are critical for in utero survival that had not yet been reported in association with stillbirth. ${ }^{4}$

Our study contributes new data as the largest and most comprehensive intergenerational study of stillbirth. It is the first to identify familial aggregation of stillbirth and quantifies stillbirth risk based on an individual's family history. Strengths of this work include use of the Utah Population Database which enabled access to a vast number of pedigrees. These genealogic records are linked to birth and fetal death certificates which allowed increased accuracy as compared to patient surveys. ${ }^{32}$ The Utah population has been shown to be of similar genetic diversity to the U.S. population of Northern European descent and thus may not be generalizable to the general U.S. population. ${ }^{33}$ In order to achieve the most rigorous definition of stillbirth possible within the UPDB, we utilized fetal death certificates. This strategy limited our identification of proband stillbirth cases to the era in which fetal death certificates were available (1978-2019), but allowed use of the most rigorous definition of stillbirth available in vital statistics data. 
Findings reported herein provide the first demonstration of familial aggregation of stillbirth. Further study of this population to evaluate pathogenic genetic variants will be an important next step in defining heritable genetic mutations. Knowledge of risks for stillbirth according to family history may also improve patient counseling and management. Establishing key genes involved in stillbirth will hopefully lead to better diagnostic tools and preventive measures.

\section{Disclosure of interests}

All authors report no conflict.

\section{Contribution to authorship}

JMP and TW equally contributed to the conception, planning and carrying out of the study. RMS, MWV, $\mathrm{AF}, \mathrm{LT}$ and $\mathrm{HC}$ had critical roles in the conception and planning of the study. HM, ZY, EG and TW had a critical role in data management, analyses and interpretation. TW, JMP, HM, MWV, LT, AQ, HC, DAE, CVA, FTA, LJ and RMS each had a critical role in interpretation of the analyses and writing the manuscript.

\section{Ethical approval}

Request for permission to use family structure data was approved by the Utah Resource for Genetic and Epidemiologic Research (RGE), the UPDB data use oversight committee. This study was approved by the Institutional Review Board of the University of Utah (IRB \#: 127960; date: 03/26/2020).

\section{Funding/support}

This work was supported by grant funding from the National Center for Advancing Translational Sciences of the National Institutes of Health (grant 1UL01TR002538). The content is solely the responsibility of the authors and does not necessarily represent the official views of the National Institutes of Health. Author TW is supported by the National Center for Advancing Translational Sciences of the National Institutes of Health (KL2 grant TR002549). Author CVA is supported, in part, by the National Heart, Lung, and Blood Institute (grant R01-HL150065) and the National Institute of Environmental Health Sciences (grant R01-ES033190). Author LBJ is supported by National Institute of General Medical Sciences (grant R35GM118335).

\section{Data sharing}

Editors and reviewers of our manuscript who need access to source data from the Utah Population Database beyond that which is contained in the manuscript for the purpose of evaluating our study may submit a request for data access to the corresponding author. The request for data access must be reviewed and approved by the Resource for Genetic and Epidemiologic Research (RGE) before access to the relevant data will be granted. Those requesting access must sign an RGE Confidentiality and Data Use Agreement.

\section{References}

1. Flenady V, Wojcieszek AM, Middleton P, Ellwood E, Erwich JJ, Coory M, Khong TY, Silver RM, Smith GCS, Boyle FM, Lawn JE, Blencowe H, Leisher SH, Gross MM, Horey D, Farrales L, Bloomfield F, McCowan L, Brown SJ, Joseph KS, Zeitlin J, Reinebrant HE, Cacciatore J, Ravaldi C, Vannacci A, Cassidy J, Cassidy P, Farquhar C, Wallace E, Siassakos D, Heazell AEP, Storey C, Sadler L, Petersen S, Frøen JF, Goldenberg RL, for The Lancet Ending Preventable Stillbirths study group, The Lancet Stillbirths In High-Income Countries Investigator Group. Stillbirths: recall to action in high-income countries. The Lancet 2016;387(10019):691-702.

2. Stillbirth Collaborative Research Network Writing Group. Causes of death among stillbirths. JAMA 2011;306(22):2459-68.

3. Reddy UM, Page GP, Saade GR, Silver RM, Vanessa R Thorsten VR, Parker CB, Pinar H, Willinger M, Stoll BJ, Heim-Hall J, Varner MW, Goldenberg RL, Bukowski R, Wapner RJ, Drews-Botsch CD, O'Brien BM, Dudley DJ, Levy B, NICHD Stillbirth Collaborative Research Network. Karyotype versus microarray testing for genetic abnormalities after stillbirth. N Engl J Med 2012;367(23):2185-93. 
4. Stanley KE, Giordano J, Thorsten V, Buchovecky C, Thomas A, Ganapathi M, Liao J, Dharmadhikari AV, Revah-Politi A, Ernst M, Lippa N, Holmes H, Povysil G, Hostyk J, Parker CB, Goldenberg R, Saade GR, Dudley DJ, Pinar H, Hogue C, Reddy UM, Silver RM, Aggarwal V, Allen AS, Wapner RJ, Goldstein DB. Causal Genetic Variants in Stillbirth. N Engl J Med 2020;383(12):1107-16.

5. Bhattacharya S, Amalraj Raja E, Ruiz Mirazo E, Campbell DM, Lee AJ, Norman JE, Bhattacharya S. Inherited predisposition to spontaneous preterm delivery. Obstet Gynecol 2010;115(6):1125-33.

6. Wu W, Witherspoon DJ, Fraser A, Witherspoon DJ, Fraser A, Clark EAS, Rogers A, Stoddard GJ, Manuck TA, Chen K, Esplin SM, Smith KR, Varner MW, Jordy LB. The heritability of gestational age in a two-million member cohort: implications for spontaneous preterm birth. Hum Genet 2015;134(7):803-8.

7. Porter TF, Fraser AM, Hunter CY, Ward RH, Varner MW. The risk of preterm birth across generations. Obstet Gynecol 1997;90(1):63-7.

8. Svensson AC, Pawitan Y, Cnattingius S, Reilly M, Lichtenstein P. Familial aggregation of small-forgestational-age births: the importance of fetal genetic effects. Am J Obstet Gynecol 2006;194(2):475-9.

9. Wikstrom A-K, Svensson T, Kieler H, Cnattingius S. Recurrence of placental dysfunction disorders across generations. Am J Obstet Gynecol 2011;205(5):454.e1-8.

10. Ananth CV, Jablonski K, Myatt L, James M. Roberts JM, Tita ATN, Leveno KJ, Reddy UM, Varner MW, Thorp JM, Mercer BM, Peaceman, Ramin SM, Carpenter MW, Samuels P, Sciscione A, Tolosa JE, Saade G, Sorokin Y, Eunice Kennedy Shriver National Institute of Child Health and Human Development Maternal-Fetal Medicine Units Network. Risk of Ischemic Placental Disease in Relation to Family History of Preeclampsia. Am J Perinatol 2019;36(6):624-31.

11. Chesley LC, Annitto JE, Cosgrove RA. The familial factor in toxemia of pregnancy. Obstet Gynecol 1968;32(3):303-11.

12. Nilsson E, Salonen Ros H, Cnattingius S, Lichtenstein P. The importance of genetic and environmental effects for pre-eclampsia and gestational hypertension: a family study. BJOG Int J Obstet Gynaecol 2004;111(3):200-6.

13. Esplin MS, Fausett MB, Fraser A, Kerber R, Mineau G, Carrillo J, Varner MW. Paternal and maternal components of the predisposition to preeclampsia. N Engl J Med 2001;344(12):867-72.

14. Woolner AMF, Raja EA, Bhattacharya S, Danielian P, Bhattacharya S. Inherited predisposition to stillbirth: an intergenerational analysis of 26,788 mother-daughter pairs. Am J Obstet Gynecol 2019;220(4):393.e1-393.e7.

15. Haig D. Genetic conflicts in human pregnancy. Q Rev Biol 1993;68(4):495-532.

16. Utah Population Database. Utah Population Database - Huntsman Cancer Institute | University of Utah. (2021, July 8). https://uofuhealth.utah.edu/huntsman/utah-population-database/.

17. Silver RM, Branch DW, Goldenberg R, Iams JD, Klebanoff MA. Nomenclature for pregnancy outcomes: time for a change. Obstet Gynecol 2011;118(6):1402-8.

18. National Center for Health Statistics (U.S.), Dundon ML, Gay GA, George JL, editors. The 1978 revision of the U.S. standard certificates. Hyattsville, Md. : Washington, D.C: U.S. Dept. of Health and Human Services, Public Health Service, National Center for Health Statistics ; For sale by the Supt. of Docs., U.S. G.P.O; 1983.

19. Guide to Completing the Facility Worksheets for the Certificate of Live Birth and Report of Fetal Death (2003 revision). [Internet]. 2006;Available from: http://www.cdc.gov/nchs/data/dvs/GuidetoCompleteFacilityWks.pdf 
20. DuVall SL, Fraser AM, Rowe K, Thomas A, Mineau GP. Evaluation of record linkage between a large healthcare provider and the Utah Population Database. J Am Med Inform Assoc JAMIA 2012;19(e1):e54-59.

21. Reddy UM, Ko C-W, Willinger M. Maternal age and the risk of stillbirth throughout pregnancy in the United States. Am J Obstet Gynecol 2006;195(3):764-70.

22. Fretts RC, Schmittdiel J, McLean FH, Usher RH, Goldman MB. Increased maternal age and the risk of fetal death. N Engl J Med 1995;333(15):953-7.

23. Kerber RA. Method for calculating risk associated with family history of a disease. Genet Epidemiol 1995;12(3):291-301.

24. Lange K, Sinsheimer JS. Calculation of genetic identity coefficients. Ann Hum Genet 1992;56(4):339-46.

25. Coon H, Darlington T, Pimentel R, Smith KR, Huff CD, Hu H, Jerominski L, Hansen J, Klein M, Callor WB, Byrd J, Bakian A, Crowell SE, McMahon WM, Rajamanickam V, Camp NJ, McGlade E, YurgelunTodd D, Grey T, Gray D. Genetic risk factors in two Utah pedigrees at high risk for suicide. Transl Psychiatry 2013;3:e325.

26. Wapner RJ, Lewis D. Genetics and metabolic causes of stillbirth. Semin Perinatol 2002;26(1):70-4.

27. Miskovic S, Culic V, Konjevoda P, Pavelic J. Positive reproductive family history for spontaneous abortion: predictor for recurrent miscarriage in young couples. Eur J Obstet Gynecol Reprod Biol 2012;161(2):182-6.

28. Pouta A, Jarvelin M-R, Hemminki E, Sovio U, Hartikainen A-L. Mothers and daughters: intergenerational patterns of reproduction. Eur J Public Health 2005;15(2):195-9.

29. Woolner AMF, Raja EA, Bhattacharya S, Danielian P, Bhattacharya S. Inherited susceptibility to miscarriage: a nested case-control study of 31,565 women from an intergenerational cohort. Am J Obstet Gynecol 2020;222(2):168.e1-168.e8.

30. Jirtle RL, Skinner MK. Environmental epigenomics and disease susceptibility. Nat Rev Genet $2007 ; 8(4): 253-62$.

31. Wilkins-Haug L. Genetic innovations and our understanding of stillbirth. Hum Genet 2020;139(9):116172.

32. Adams MM, Wilson HG, Casto DL, Berg CJ, McDermott JM, Gaudino JA, McCarthy BJ. Constructing reproductive histories by linking vital records. Am J Epidemiol 1997;145(4):339-48.

33. McLellan T, Jorde LB, Skolnick MH. Genetic distances between the Utah Mormons and related populations. Am J Hum Genet 1984;36(4):836-57.

Table 1 . The top 10 high-risk pedigrees with statistically significant evidence for familial aggregation of stillbirth.

\begin{tabular}{llllllll}
\hline Pedigree & Birth Year $^{\mathbf{2}}$ & Death Year $^{\mathbf{2}}$ & Total Descendants & FSIR $^{\mathbf{3}}$ & FSIR P-value $^{\mathbf{3}}$ & Observed $^{\mathbf{4}}$ & Expectec \\
\hline 1 & 1782 & NA & 813 & 7.54 & $<0.001$ & 5 & 1 \\
2 & 1843 & 1908 & 1044 & 6.48 & $<0.001$ & 45 & 7 \\
3 & 1815 & 1893 & 552 & 6.32 & $<0.001$ & 13 & 2 \\
4 & 1865 & 1928 & 1587 & 6.22 & $<0.001$ & 119 & 19 \\
5 & 1864 & 1935 & 1491 & 5.25 & $<0.001$ & 41 & 8 \\
6 & 1836 & 1902 & 703 & 5.04 & $<0.001$ & 20 & 4 \\
7 & 1857 & 1940 & 698 & 5.03 & $<0.001$ & 31 & 6 \\
8 & 1807 & 1872 & 780 & 5.01 & $<0.001$ & 15 & 3 \\
9 & 1837 & 1887 & 3655 & 4.66 & $<0.001$ & 65 & 14 \\
10 & 1838 & 1898 & 1147 & 4.61 & $<0.001$ & 34 & 7 \\
\hline
\end{tabular}


1 Top 10 pedigrees of stillbirth high-risk pedigrees $(n=390)$ of stillbirth cases $(n=2498)$ sorted by their FSIR p-values, FSIR values and founders to eliminate any multiple founders claiming the same descendants through marriage.

${ }^{2}$ Birth/death year of the founder

${ }^{3}$ Familial Standardized Incidence Ratio, comparing the incidence of stillbirth in a family to its expected incidence in the population

${ }^{4}$ The observed and expected counts of stillbirth between pedigrees may overlap

Figure 1. A pedigree (pedigree 3 in Table 1 ; FSIR $=6.32$ [p-value $<0.001]$ ) showing 10 stillbirth cases indicated in black shades. Gray circles indicate study eligible mothers. The small triangle denotes the founder. Pedigree trimmed to demonstrate the affected lineages.

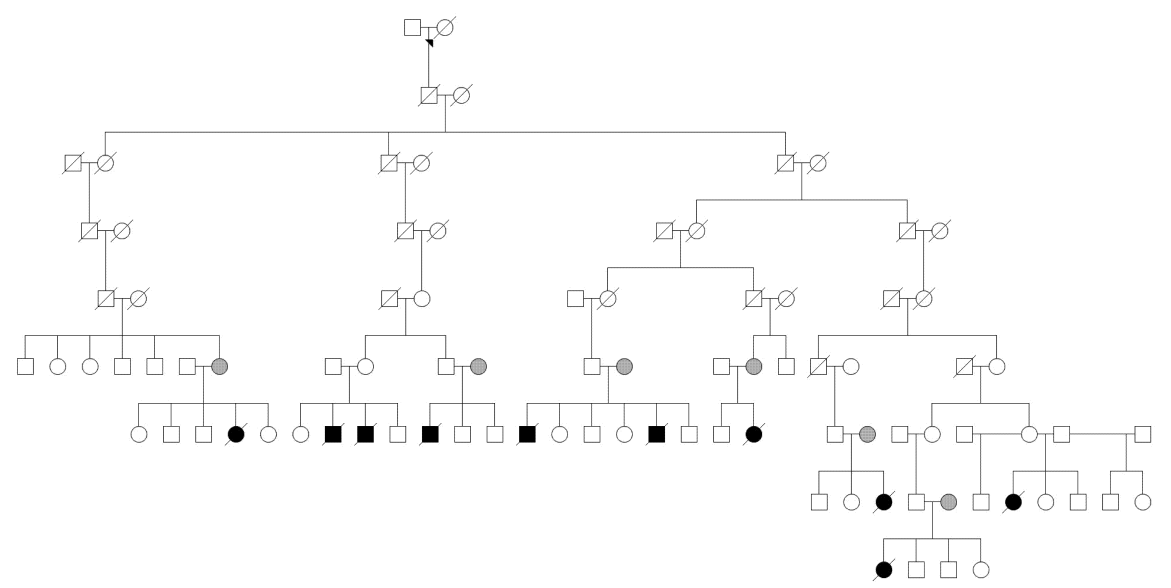

Figure 2 . Stillbirth odds among relatives of parents affected by stillbirth. 


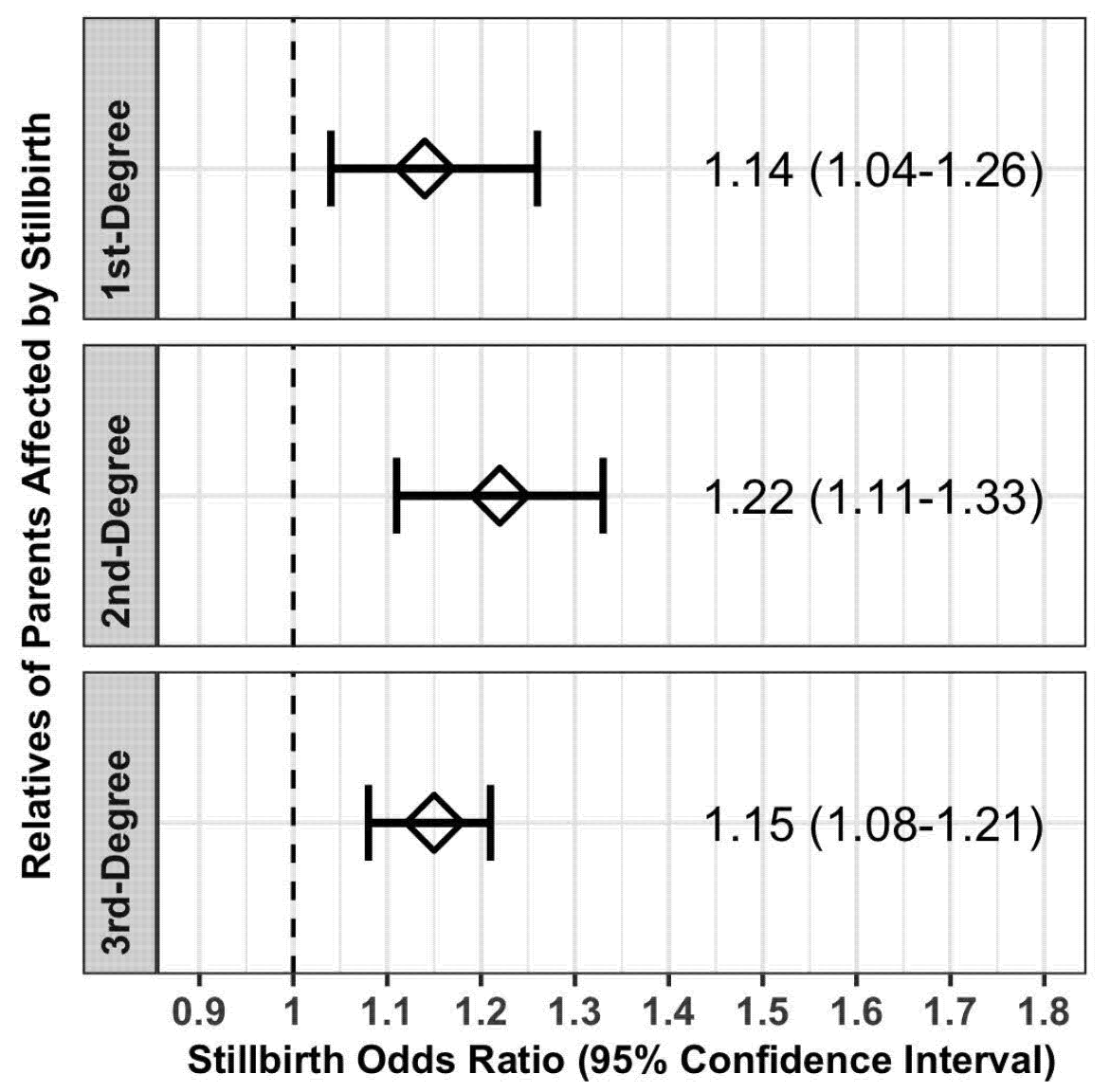

Table 2 . Stillbirth odds among relatives of parents affected by stillbirth, stratified by parental sex

\begin{tabular}{lllllll}
\hline Relationship & Affected Fathers & Affected Fathers & Affected Fathers & Affected Mothers & Affected Mothers & Affected \\
\hline & $\mathrm{N}_{\text {relatives }}$ & OR $(95 \% \mathrm{CI})$ & $\mathrm{p}$-value & $\mathrm{N}_{\text {relatives }}$ & OR $(95 \%$ CI $)$ & p-value \\
First-degree & 35,538 & $1.17(1.02,1.34)$ & 0.028 & 37,796 & $1.12(0.98,1.28)$ & 0.103 \\
Male & 19,092 & $1.22(1.02,1.47)$ & 0.033 & 19,957 & $1.05(0.86,1.28)$ & 0.632 \\
Female & 16,446 & $1.10(0.90,1.35)$ & 0.339 & 17,839 & $1.19(0.98,1.43)$ & 0.073 \\
Second-degree & 64,769 & $1.34(1.19,1.51)$ & $<0.001$ & 63,285 & $1.09(0.96,1.24)$ & 0.164 \\
Male & 40,075 & $1.38(1.17,1.62)$ & $<0.001$ & 38,545 & $1.19(0.99,1.42)$ & 0.061 \\
Female & 24,694 & $1.31(1.10,1.57)$ & 0.003 & 24,740 & $1.01(0.84,1.21)$ & 0.942 \\
Third-degree & 153,765 & $1.14(1.06,1.24)$ & 0.001 & 146,718 & $1.15(1.06,1.24)$ & 0.001 \\
Male & 102,769 & $1.17(1.05,1.30)$ & 0.004 & 97,618 & $1.17(1.05,1.31)$ & 0.005 \\
Female & 50,996 & $1.12(1.00,1.25)$ & 0.048 & 49,100 & $1.12(1.00,1.25)$ & 0.044 \\
\hline
\end{tabular}

Table S1 : Demographic characteristics of stillbirth cases and controls.

\begin{tabular}{lll}
\hline & Stillbirth Cases & Controls \\
\hline $\begin{array}{l}\text { Maternal Demographic and Clinical Characteristics } \\
\text { Age at index stillbirth/birth, years [mean (SD)] }\end{array}$ & $(\mathrm{N}=9404)$ & $(\mathrm{N}=18808)$ \\
Race/ethnicity & $27.5 \pm 5.8$ & $27.5 \pm 5.8$
\end{tabular}




\begin{tabular}{|c|c|c|}
\hline & Stillbirth Cases & Controls \\
\hline \multicolumn{3}{|l|}{ Non-Hispanic } \\
\hline American Indian or Alaska Native & $61(0.6 \%)$ & $101(0.5 \%)$ \\
\hline Asian & $61(0.6 \%)$ & $153(0.8 \%)$ \\
\hline Native Hawaiian or Other Pacific Islander & $51(0.5 \%)$ & $52(0.3 \%)$ \\
\hline Black or African American & $62(0.7 \%)$ & $51(0.3 \%)$ \\
\hline White & $6204(66.0 \%)$ & $13731(73.0 \%)$ \\
\hline Multiple Races & $442(4.7 \%)$ & $692(3.7 \%)$ \\
\hline Hispanic & $2301(24.5 \%)$ & $3994(21.2 \%)$ \\
\hline Unknown race/ethnicity & $222(2.4 \%)$ & $34(0.2 \%)$ \\
\hline Mother's birth year & $1971.5 \pm 12.9$ & $1971.5 \pm 12.9$ \\
\hline \multicolumn{3}{|l|}{ Mother's education at index birth } \\
\hline High school degree or less & $4046(43.0 \%)$ & $7617(40.5 \%)$ \\
\hline Some college and college degree & $4269(45.4 \%)$ & $9638(51.2 \%)$ \\
\hline Post-college & $424(4.5 \%)$ & $1340(7.1 \%)$ \\
\hline Unknown & $665(7.1 \%)$ & $213(1.1 \%)$ \\
\hline Number of prenatal visits ${ }^{2}$ & $6(4-9)$ & $11(9-12)$ \\
\hline Number of previous live births ${ }^{2}$ & $1(0-2)$ & $1(0-2)$ \\
\hline At least one previous live birth still living & $5473(58.2 \%)$ & $12073(64.2 \%)$ \\
\hline \multicolumn{3}{|l|}{ Method of delivery } \\
\hline Cesarean and vaginal/forceps/vacuum & $0(0.0 \%)$ & $27(0.1 \%)$ \\
\hline Cesarean only & $678(7.2 \%)$ & $3495(18.6 \%)$ \\
\hline Vaginal/forceps/vacuum only & $7310(77.7 \%)$ & $15216(80.9 \%)$ \\
\hline Others/Unknown & $1416(15.1 \%)$ & $70(0.4 \%)$ \\
\hline Had at least one birth defect & $686(7.3 \%)$ & $159(0.8 \%)$ \\
\hline Maternal Pre-Pregnancy Body Mass Index $\left(\mathrm{BMI}, \mathrm{kg} / \mathrm{m}^{2}\right)^{2}$ & $24.0(21.1-29.1)$ & $23.2(20.7-27.4)$ \\
\hline Missing maternal pre-pregnancy BMI & $3477(37.0 \%)$ & $5592(29.7 \%)$ \\
\hline Mother's weight gain during index pregnancy $(\mathrm{kg})^{2}$ & $8.2(4.1-12.7)$ & $13.6(10.4-18.1)$ \\
\hline Missing mother's weight gain due to index pregnancy & $3349(35.6 \%)$ & $5383(28.6 \%)$ \\
\hline Mother had gestational diabetes due to index pregnancy & $141(1.5 \%)$ & $384(2.0 \%)$ \\
\hline Mother had pre-existing diabetes prior to index pregnancy & $152(1.6 \%)$ & $110(0.6 \%)$ \\
\hline Mother had diabetes (gestational/pre-existing) during index pregnancy & $302(3.2 \%)$ & $498(2.6 \%)$ \\
\hline Mother had gestational hypertension due to index pregnancy & $1166(12.4 \%)$ & $1861(9.9 \%)$ \\
\hline Mother had chronic hypertension prior to index pregnancy & $95(1.0 \%)$ & $115(0.6 \%)$ \\
\hline \multicolumn{3}{|l|}{ Paternal Demographic and Clinical Characteristics } \\
\hline Age at index stillbirth/birth, years & $30.3 \pm 6.4$ & $30.1 \pm 6.2$ \\
\hline \multicolumn{3}{|l|}{ Race/ethnicity } \\
\hline \multicolumn{3}{|l|}{ Non-Hispanic } \\
\hline American Indian or Alaska Native & $50(0.5 \%)$ & $59(0.3 \%)$ \\
\hline Asian & $48(0.5 \%)$ & $105(0.6 \%)$ \\
\hline Native Hawaiian or Other Pacific Islander & $39(0.4 \%)$ & $73(0.4 \%)$ \\
\hline Black or African American & $71(0.8 \%)$ & $87(0.5 \%)$ \\
\hline White & $5702(60.6 \%)$ & $13109(69.7 \%)$ \\
\hline Multiple Races & $276(2.9 \%)$ & $640(3.4 \%)$ \\
\hline Hispanic & $1517(16.1 \%)$ & $3132(16.7 \%)$ \\
\hline Unknown & $1701(18.1 \%)$ & $1603(8.5 \%)$ \\
\hline Father's birth year & $1968.6 \pm 13.4$ & $1969.0 \pm 13.2$ \\
\hline \multicolumn{3}{|l|}{ Father's education at index birth } \\
\hline High school degree or less & $1891(20.1 \%)$ & $5904(31.4 \%)$ \\
\hline Some college and college degree & $1992(21.2 \%)$ & $8729(46.4 \%)$ \\
\hline Post-college & $605(6.4 \%)$ & $2641(14.0 \%)$ \\
\hline
\end{tabular}




\begin{tabular}{lll}
\hline & Stillbirth Cases & Controls \\
\hline Unknown $^{5}$ & $4916(52.3 \%)$ & $1534(8.2 \%)$ \\
Index Stillborn/Live-born Demographic and Clinical Characteristics & & \\
Gestational Age, weeks & & \\
Sex & $29(23-36)$ & $39(38-40)$ \\
Male & $4735(50.4 \%)$ & $9778(52.0 \%)$ \\
Female & $4526(48.1 \%)$ & $9030(48.0 \%)$ \\
Birth year & $1999.0 \pm 12.5$ & $1999.0 \pm 12.5$ \\
Any fetal structural anomalies or birth defects & $1686(7.3 \%)$ & $159(0.8 \%)$ \\
Birthweight, g & $1145(440-2567)$ & $3340(3020-3646)$ \\
Any Stillbirth Family History & & \\
All relatives & $912(12.5 \%)$ & $1524(11.6 \%)$ \\
First-degree relatives & $215(3 \%)$ & $387(2.9 \%)$ \\
Second-degree relatives & $277(3.8 \%)$ & $416(3.2 \%)$ \\
Third-degree relatives & $563(7.7 \%)$ & $911(6.9 \%)$ \\
\hline
\end{tabular}

${ }^{1}$ Demographic characteristics of cases and controls were compared using t-tests for continuous variables and chi-squared tests for categorical variables.

${ }^{2}$ Values are median and inter-quartile range and exclude missing values

${ }^{3}$ There were 9 live births with birth weight $(\mathrm{g})<500$. The accepted range for birth weights of live births are $500-6000 \mathrm{~g}$.

${ }^{4}$ Difference in education status is due to a reduced rate of maternal form completion in fetal death cases as compared to controls.

${ }^{5}$ This difference is due to 1) father's education was available for all years during the period 1978-2016 from birth certificates; but father's education was only available for the years 1978-2003 from fetal death certificates. 2) fathers are less likely reported in fetal death certificates

Figure S1 . STROBE diagram showing inclusion and exclusion criteria for relatives of parents of stillbirth cases and controls.

\section{Hosted file}

image3.emf available at https://authorea.com/users/460174/articles/557071-familial-

aggregation-of-stillbirth-a-pedigree-analysis-of-a-matched-case-control-study

Figure S2 . Distribution of familial standardized incidence ratio values among stillbirth high-risk pedigrees. 


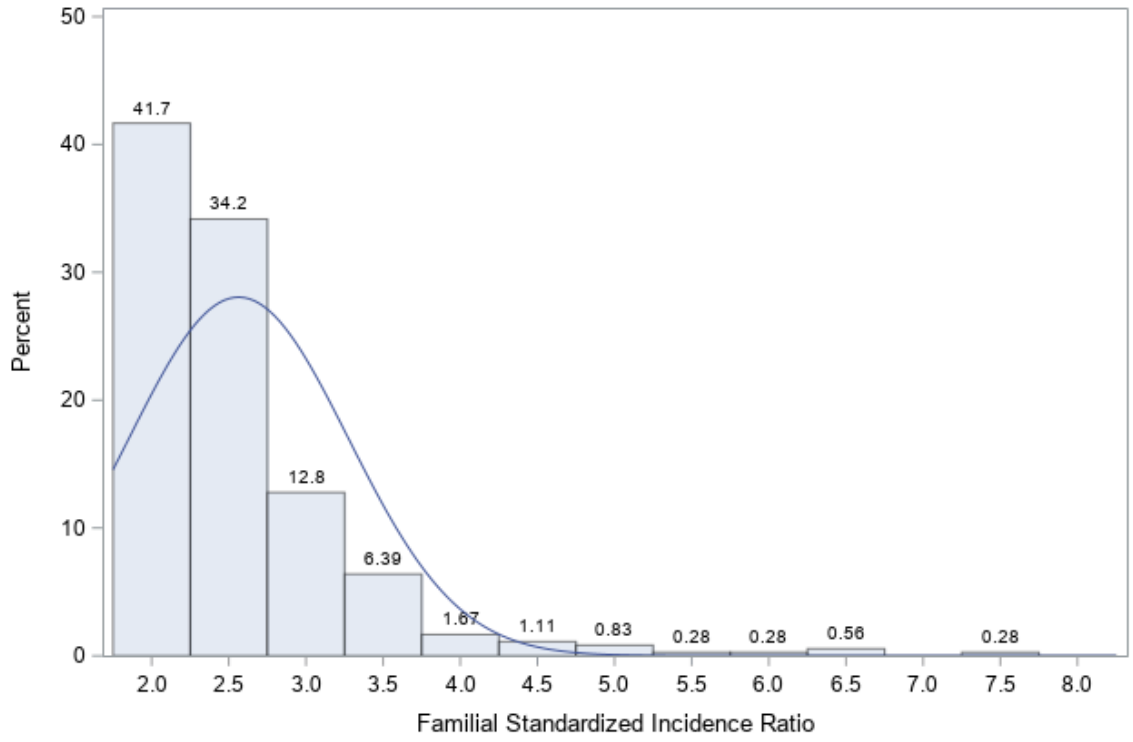

